\title{
Effects of the Location of Myocardial Infarction on the Spectral Characteristics of Ventricular Fibrillation
}

\author{
JUAN J. SÁNCHEZ-MUÑOZ, M.D., Ph.D., * JOSÉ L. ROJO-ÁLVAREZ, PH.D.,† \\ ARCADIO GARCÍA-ALBEROLA, M.D. Ph.D., * ESTRELLA EVERSS, PH.D.,† \\ JESÚS REQUENA-CARRIÓN,† MERCEDES ORTIZ, PH.D.„‡ FELIPE ALONSO-ATIENZA,† \\ and MARIANO VALDÉS-CHAVARRI, M.D. PH.D.* \\ From the * Arrhythmia Unit, Hospital Universitario Virgen de la Arrixaca de Murcia, Murcia, Spain, tDepartment of \\ Signal Theory and Communications, University Rey Juan Carlos, Madrid, Spain, and ¥Arrhythmia Unit, Hospital \\ General Universitario Gregorio Marañón, Madrid, Spain
}

Background: The location of the myocardial infarction (MI) might modify the spectral characteristics of ventricular fibrillation (VF) in humans.

Objective: To evaluate the effect of the location of the infarcted area on the spectral parameters of VF.

Methods: Patients with chronic MI (29 anterior, 32 inferior) and induced VF during cardioverter defibrillator implant were retrospectively studied. Dominant frequency $\left(f_{d}\right)$, organization index $(O I)$, and power of the harmonic peaks were calculated in the device-stored electrograms (EGM) during sinus rhythm (SR) and $V F$.

Results: The $f_{d}$ of the VF was not affected by the left ventricular ejection fraction (LVEF) or the MI location (anterior: $4.54 \pm 0.74 \mathrm{~Hz}$, inferior: $4.77 \pm 0.48 \mathrm{~Hz}$, n.s.). The OI was also similar in both groups. However, in patients with inferior MIs, normalized peak power at $f_{d}$ was higher (118.3 \pm 18.5 vs $100.6 \pm$ 28.2, $P<0.01)$ and the normalized peak power of the harmonics was lower than in the anterior MI group. The analysis of EGM during SR showed similar results. The size of the necrotic area and its distance to the recording electrode might partially explain these results.

Conclusion: In our series, the spectral characteristics of the EGMs during VF showed significant differences depending on the MI localization. A higher fraction of energy (in the low-frequency region) was seen in inferior MIs, whereas the peak power at the harmonics increased in anterior MIs. A similar effect was seen during SR and VF, suggesting that it is caused by local electrophysiology abnormalities induced by the MI rather than by different intrinsic characteristics of the VF. (PACE 2008; 31:660-665)

myocardial infarction, ventricular fibrillation, spectral analysis, signal analysis, ICD, electrogram.

\section{Introduction}

Electrical activity during ventricular fibrillation (VF) has been considered as a highly disorganized and random process. However, the spectral analysis of optical or electrical recordings during VF reveals a substantial degree of structure and organization, and it allows the estimation of several periodicity and regularity indexes. ${ }^{1-3}$ Spectral VF parameters have been related to the duration of the arrhythmia, ${ }^{4}$ the presence of ischemia, ${ }^{5}$ the action of antiarrhythmic drugs, ${ }^{6}$ or the outcome of shock therapy. ${ }^{7}$ The existence of a necrotic area in the myocardium has been shown to alter the frequency spectrum in a dog model of VF. ${ }^{8}$ Moreover, the maintenance and characteristics of the VF may be related to mother rotors anchored to

Potential conflicts of interest: None

Address for reprints: Arcadio García-Alberola, M.D., Ph.D., Arrhythmia Unit, Hospital Virgen de la Arrixaca, Ctra Cartagena s/n, km 20 30120-El Palmar, Murcia, Spain. Fax: +34968883036; e-mail: arcadi@secardiologia.es

Received October 25, 2007; revised January 3 2008; accepted January24, 2008. specific anatomic structures, such as the papillary muscles, that can be affected by infarcted areas. ${ }^{9}$ Very little information is available on the spectral characteristics of the VF in patients with chronic myocardial infarction (MI). Specifically, it is not known whether the spectrum of this VF depends on the infarct location. The objectives of this study are to describe the characteristics of the electrical signal recorded by an intracardiac electrode during induced VF in patients with chronic MI, and to evaluate the effect of the location of the infarcted area on the VF spectral features.

\section{Methods}

Consecutive patients with chronic MI > 3 months old undergoing an implantable cardioverter defibrillator (ICD) implant for clinical reasons between November 2001 and August 2003 in two tertiary hospitals were included in this retrospective study. Clinical data as well as the location of the infarct were obtained from the clinical reports. The left ventricular ejection fraction (LVEF) was categorized in four degrees, from normal ( $>0.55)$ to slightly ( 0.41 to 0.55$)$, moderately

(C)2008, The Authors. Journal compilation (C)2008, Blackwell Publishing, Inc. 
(0.31 to 0.40$)$, or severely depressed $(<0.31)$. The implant protocol at the time of the study required the induction of at least two consecutive $\mathrm{VF}$ episodes in order to ensure a safety margin for defibrillation. The ICD was programmed to record intracardiac electrograms (EGMs) during VF in a pseudounipolar configuration using the defibrillation coil in the right ventricle and the can as the indifferent electrode. The recorded EGMs were saved in digital format (128 samples per second) for further analysis.

\section{VF Signal Processing and Spectral Analysis}

The onset of each VF episode was manually annotated, and the following 3 seconds were selected for processing. We decided to analyze the first 3 seconds because this period of time was deemed to be adequate to estimate the spectrum of the signal according to the sampling frequency and the spectral band of interest. An automatic cancellation of low-frequency baseline oscillations (trends) was implemented using a 250-ms median filter and spline interpolation. ${ }^{10}$ A spectral representation $\mathrm{P}(\mathrm{f})$ was obtained for each EGM segment with a Welch periodogram, with Hamming window of 128 samples, $50 \%$ overlap, and spectral resolution of 1,024 samples. This spectral representation was normalized to unit area, which is denoted as $\mathrm{P}_{\mathrm{n}}(\mathrm{f})$. In many cases, spectral profile of the monopolar EGMs closely corresponded to a harmonic structure, which was taken into account for using conventional parameters in this setting. These spectral parameters and its meaning have been recently reviewed, ${ }^{11}$ and they are briefly summarized next (see Fig. 1):

- Dominant Frequency ( $f d$ ): It is the frequency at which the absolute spectral maximum occurs.

- Fundamental Frequency ( $f_{0}$ ): For periodic signals, it is the inverse of the cycle length, that is, the inverse of the minimum repetition period.

- Harmonics and Power Peaks: For periodic signals, harmonics are the frequencies corresponding to the integer multiples of fundamental frequency $\mathrm{f}_{0}$. We considered up to five harmonics, given by $f_{2}=2 f_{0}$ to $f_{5}=5 f_{0}$. When the power peak did not happen exactly at the harmonic frequency due to noise, it was adjusted to correspond to the nearest relative maximum, within a tolerance of about $1.5 \mathrm{~Hz}$. Accordingly, we measured the peaks of the normalized power spectrum at each frequency, denoted by $P_{n}\left(f_{d}\right), P_{n}\left(f_{0}\right)$, and $P_{n}\left(f_{2}\right)$ to $\mathrm{P}_{\mathrm{n}}\left(\mathrm{f}_{5}\right)$. The obtained values were multiplied by a factor of 1,000 in order to make the numerical expressions easier.

- Organization Index: We defined the Organization Index (OI) as follows. Given that the EGM spectra were mostly contained in the band from 4
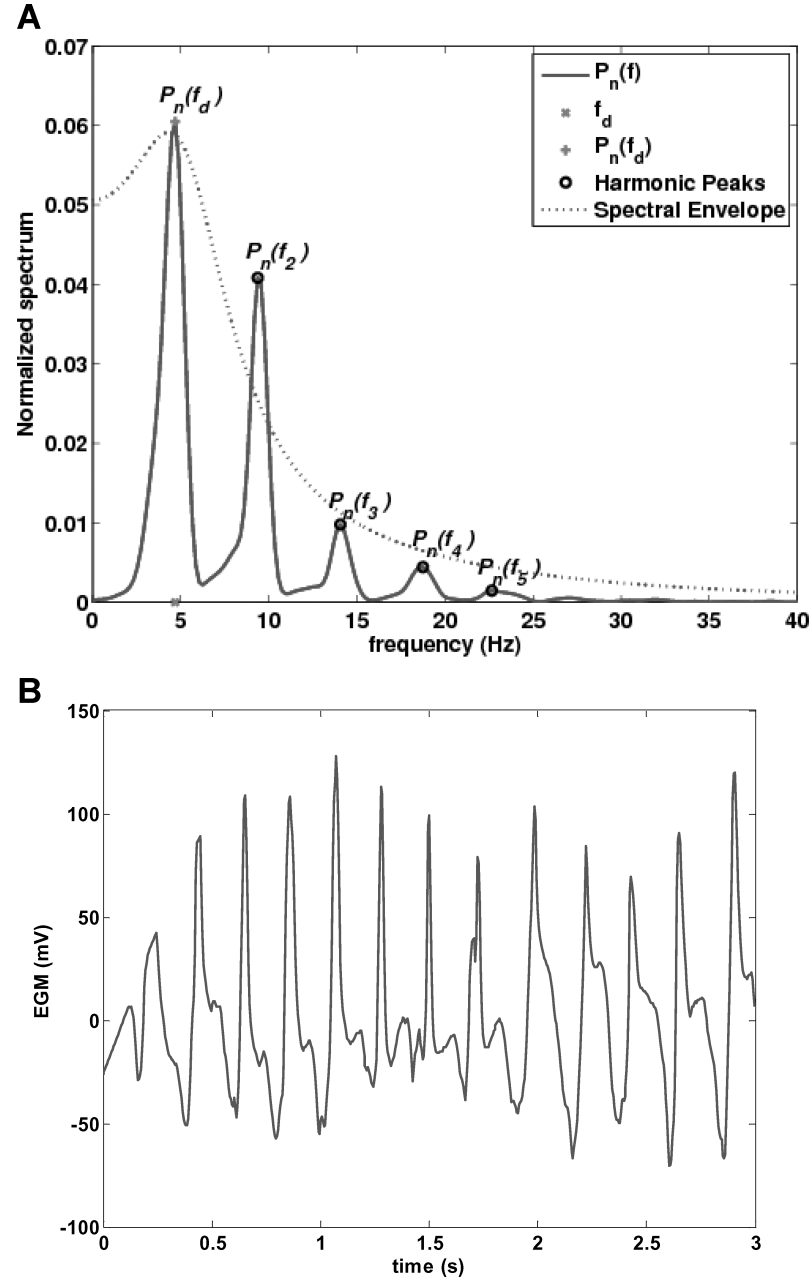

Figure 1. An ICD-stored EGM VF example. (A) Spectral parameters measured from the power spectrum and spectral envelope. (B) Monopolar EGM.

to $30 \mathrm{~Hz}$, we first obtained the total power in this band. Then, for the spectral peaks corresponding to fundamental frequency $f_{0}$ and to every harmonic peak within this band, their power in a bandwidth given by the $75 \%$ decrease in amplitude in each peak was obtained and summed. Accordingly, the OI was calculated as the ratio between the power in the bandwidth of $\mathrm{f}_{0}$ and its spectral harmonics, and the total power in the band from 4 to $30 \mathrm{~Hz} .{ }^{12}$

- Spectral Envelope: In order to yield an independent from periodicity spectral characterization, an auto-regressive model (Yule-Walker method, fourth order) was fitted for each EGM signal during $\mathrm{VF}$, and the squared spectral envelope was estimated, thus yielding a spectral representation that has no harmonic structure, and hence it contains no explicit information about the periodicity, but rather about the underlying 
physiological phenomena and abut the acquisition characteristics. $^{13}$

\section{SR Signal Processing and Spectral Analysis}

In order to evaluate whether the differences in the VF spectrum between the study groups represent a specific behavior of the VF for each infarct location or they just reflect intrinsic local electrophysiologic properties of the myocardium related to the infarcted area, the frequency content of the EGM during sinus rhythm (SR) was also analyzed. For this purpose, we obtained the ICD-stored EGM of SR for each patient. Spectral envelope was also estimated for each SR recording.

\section{Simulations}

To reproduce and to explain the results obtained in the previous analysis, a simulation study was conducted. The model has been described in detail elsewhere. ${ }^{14}$ In brief, it consists of a cellular automaton model, with activation being governed by a static conventional restitution curve. Additionally, an EGM recording model is given by the volume conductor equation in a homogeneous medium, and it was tuned for modeling a simple unipolar electrode configuration. As shown in Figure 2, a $1 \times 2$-cm cellular tissue sheet was discretized (80 cell groups per $\mathrm{cm}$ ). We compared homogeneous tissue with the presence of an infarcted, electrically silent region. Simulated EGMs were obtained at two different locations, as described in Figure 2. The infarcted region was considered either to the left, according to the figure, or to the right of the sheet, allowing us to analyze the effect of proximity between the infarct and the electrode. Unipolar EGMs were recorded at $\mathrm{x}=$

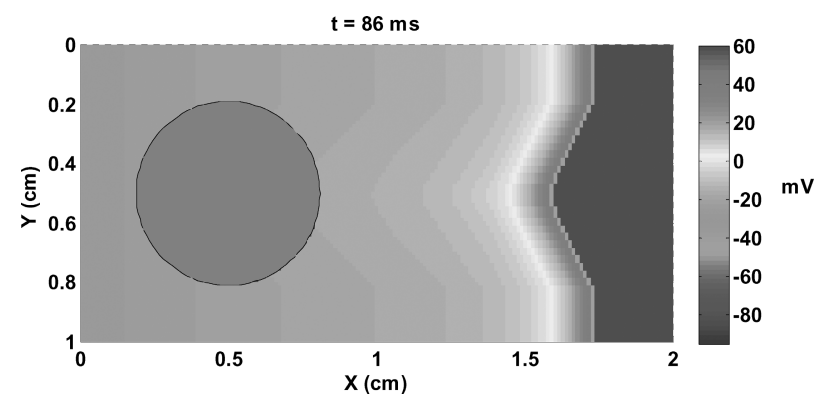

Figure 2. Simulation model. Grey zone represents the infarcted region, and it was considered either to the left, according to the figure, or to the right of the sheet, to analyze the effect of proximity between the infarct and the electrode. Unipolar EGMs were recorded at $x=2.4 \mathrm{~cm}$ and $y=0.5 \mathrm{~cm}$, at a vertical distance to the plane of $0.2 \mathrm{~cm}$. To analyze the effect of distance, an electrode was also modeled at $\mathrm{x}=4 \mathrm{~cm}$.
$2.4 \mathrm{~cm}$ and $\mathrm{y}=0.5 \mathrm{~cm}$, at a vertical distance to the plane of $0.2 \mathrm{~cm}$. In order to analyze the effect of distance, an electrode was also modeled at $\mathrm{x}=$ $4 \mathrm{~cm}$ and $\mathrm{y}=0.5 \mathrm{~cm}$ at the same vertical distance.

\section{Results}

Sixty-one patients were included in the study, 29 with anterior MI (47.5\%, group A) and 32 with inferior MI (52.5\%, group B). Age (63.0 \pm 9.9 years for group A, $65.2 \pm 8.6$ years for group B) and gender (one female in each group) were not significantly different between groups. An LVEF $<30 \%$ was observed in $22 / 29(75.9 \%)$ patients with anterior $\mathrm{MI}$ and in 11/32 (34.4\%) patients of group $\mathrm{B}$ $(\mathrm{P}=0.001)$. The spectral parameters were not affected by the degree of left ventricular dysfunction. Specifically, the dominant frequency did not show significant differences between patients with LVEF $<0.30(4.61 \pm 0.69 \mathrm{~Hz})$ and patients with LVEF $>$ $0.30(4.71 \pm 0.55 \mathrm{~Hz}, \mathrm{n} . \mathrm{s}$.) and similar results were obtained for $\mathrm{P}_{\mathrm{n}}\left(\mathrm{f}_{\mathrm{d}}\right)(0.12 \pm 0.03$ vs $0.11 \pm 0.02)$ and OI $(0.79 \pm 0.09$ vs $0.78 \pm 0.07$, respectively, both nonsignificant).

Low correlation coefficients were obtained for the spectral variables ( $R$ of 0.47 for $f_{d}, 0.70$ for $f_{0}$, 0.01 for $P_{n}\left(f_{d}\right), 0.01$ for $P_{n}\left(f_{2}\right), 0.18$ for $P_{n}\left(f_{3}\right), 0.22$ for $P_{n}\left(f_{4}\right), 0.15$ for $P_{n}\left(f_{5}\right)$, and 0.19 for OI). Therefore, each measured spectral parameter was averaged in the two consecutive VF episodes, when available.

\section{VF Signal Processing and Spectral Analysis}

The $f_{d}$ was similar for patients with anterior and inferior MIs $(4.54 \pm 0.75 \mathrm{~Hz}$ and $4.77 \pm$ $0.49 \mathrm{~Hz}$, respectively, n.s.). The differences in $\mathrm{f}_{0}$ and OI were also nonsignificant. In contrast, the spectral pattern in groups A and B was significantly different (see Table I). In patients with inferior MI, normalized power at the $f_{0}$ peak was significantly higher $(0.10 \pm 0.02$ vs $0.11 \pm 0.01$,

\section{Table I.}

Spectral Characteristics of the VF Signal in Anterior and Inferior Infarcts

\begin{tabular}{lccc}
\hline & Anterior & Inferior & P Value \\
\hline $\mathrm{f}_{d}$ & $4.54 \pm 0.74$ & $4.77 \pm 0.48$ & n.s. \\
$\mathrm{f}_{0}$ & $4.54 \pm 0.75$ & $4.76 \pm 0.47$ & n.s. \\
$\mathrm{P}_{\mathrm{n}}\left(\mathrm{f}_{\mathrm{d}}\right)$ & $100.6 \pm 28.2$ & $118.3 \pm 18.5$ & 0.006 \\
$\mathrm{P}_{\mathrm{n}}\left(\mathrm{f}_{\mathrm{f}}\right)$ & $20.0 \pm 10.3$ & $13.7 \pm 7.9$ & 0.01 \\
$\mathrm{P}_{\mathrm{n}}\left(\mathrm{f}_{3}\right)$ & $5.4 \pm 3.8$ & $2.9 \pm 2.5$ & 0.004 \\
$\mathrm{P}_{\mathrm{n}}\left(\mathrm{f}_{4}\right)$ & $1.8 \pm 1.7$ & $0.9 \pm 0.9$ & 0.012 \\
$\mathrm{P}_{\mathrm{n}}\left(\mathrm{f}_{5}\right)$ & $0.8 \pm 0.9$ & $0.3 \pm 0.2$ & 0.003 \\
$\mathrm{Ol}$ & $0.79 \pm 0.12$ & $0.80 \pm 0.07$ & n.s. \\
\hline
\end{tabular}


A

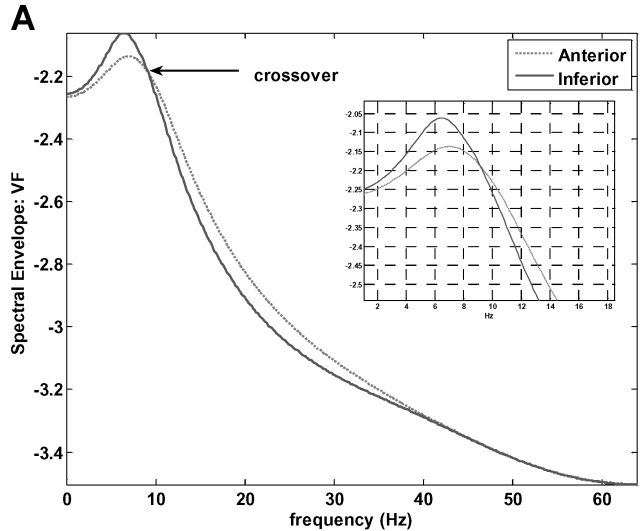

C

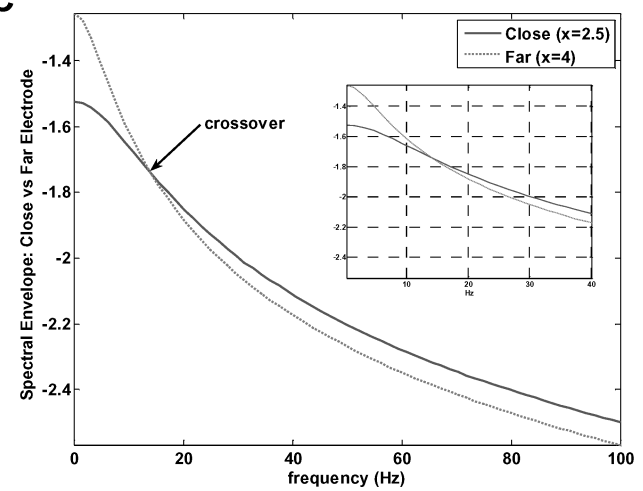

B

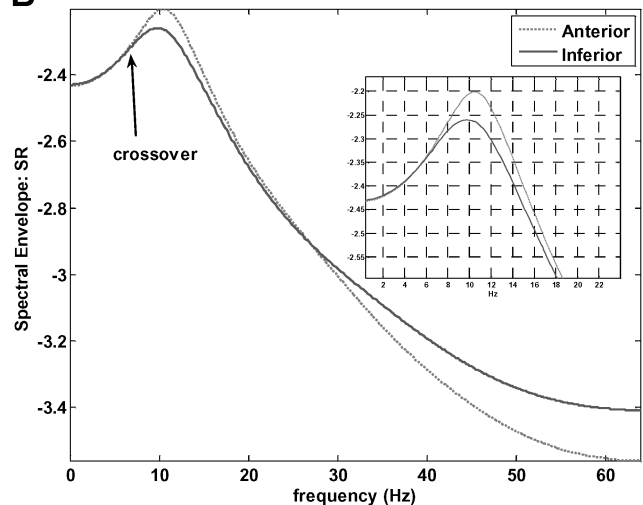

D

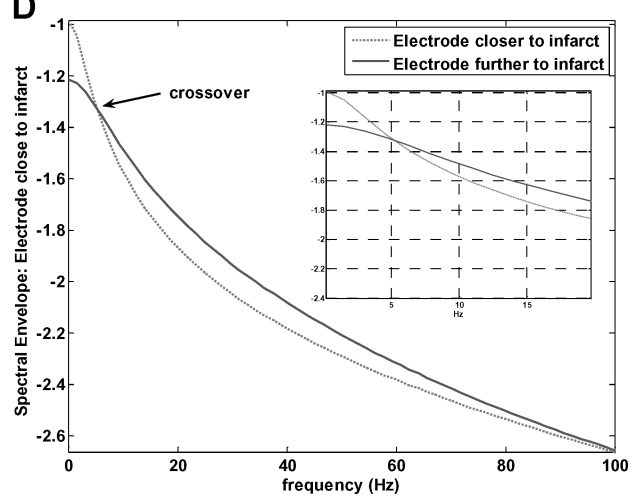

Figure 3. Averaged spectral envelopes in logarithmic units and crossover about $10 \mathrm{~Hz}$. (A) VF in ICD-stored EGM. (B) SR in ICD-stored EGM. (C) Simulation of changes with distance. (D) Simulations of changes with proximity between electrode and infarction.

$\mathrm{P}<0.01$ ), whereas the normalized spectral power at the harmonics was higher in patients with anterior MI. The same analysis did not yield any significant difference when made on tip-to-coil configuration available in the ICD-stored EGM, and hence, those results are not included here for shortness. Also, the analysis of the first 4 or 5 seconds gave similar results (not included). The averaged spectra in logarithmic units for both groups are depicted in Figure 3. A crossover of the spectral envelopes is clearly seen, with greater fractional power below $10 \mathrm{~Hz}$ approximately in inferior infarctions, and showing a significant, opposite trend above this frequency. The spectral parameters were not affected by the degree of left ventricular dysfunction.

\section{SR Signal Processing and Spectral Analysis}

The averaged spectral envelopes in logarithmic units for both groups during SR are shown in Figure 3. A crossover between spectral envelopes at about $10 \mathrm{~Hz}$ is again apparent, showing a lowpass filtering effect for inferior MIs, very similar to the one observed in the preceding VF analysis.
This crossover was also obtained using instead an autoregressive spectral analysis (not shown) thus demonstrating that it was not a phenomenon dependent on the signal processing fitting procedure. The use of the same electrode configuration for the acquisition of the EGMs during VF and SR may contribute to explain the similarity of both spectral envelopes as observed in Figs. 3A and B.

\section{Simulations}

Possible reasons for this effect were assessed using a simulation model. As shown in Figure 3, the higher frequencies become attenuated as the electrically silent area and the recording electrode come closer, thus moving the electric source away from the electrode. This low-pass filtering effect is a consequence of the electric field characteristics in quasi-electrostatic conditions. ${ }^{15}$ The crossover effect, also reproduced by the simulations, is due to the normalization of the area under the spectral curve to one. The increment of the size of the infarcted region has also a low-pass filtering effect: the greater the size of the infarction (and hence, the lesser effective myocardial mass to contribute 
to the EGM voltage), the lower the spectral content at high frequency in the unipolar EGM.

\section{Discussion}

Our study shows that the spectral characteristics of the electrical signal during induced VF recorded with an intracardiac electrode depend on the location of the infarct. In patients with inferior MIs, the contribution of the low-frequency band to the total energy of the spectrum is relatively higher, whereas the relative amplitude of the harmonics increases in patients with anterior infarcts. This effect may be due to an alteration of the electrophysiologic properties of the myocardium induced by the infarcted area and does not necessarily reflect different intrinsic characteristics of the VF. The activation mathematical model suggests that differences in the distance from the electrode to the infarcted region or in the size of this region could play a role in reducing the fractional energy of the high-frequency band in inferior MIs.

Several experimental studies have shown that the existence of an infarcted area may change the frequency content of local EGMs during VF. In a canine model of chronic MI, the cycle length of VF was longer and the degree of organization greater than in the control group. ${ }^{16}$ In a similar experimental preparation, Jacobson et al. ${ }^{8}$ found no significant changes in the $f_{d}$ or in the fractional peak power at the $f_{d}$ peak, but demonstrated a significant reduction of the total and peak power in the infarcted animals. Finally, frequency maps of the left ventricle in sheep with chronic anterior MI showed regions of high-frequency activation usually being located close to the interventricular septum, whereas a low $f_{d}$ was recorded within the infarcted area. ${ }^{17}$ However, none of these studies analyze specifically the power distribution along the frequency axis. Moreover, the effect of the infarct location on the VF characteristics cannot be assessed since the infarct is always produced by occlusion of the proximal left anterior descending coronary artery. The distribution of the necrotic area in the myocardium might potentially alter the VF spectrum by several mechanisms. First, it has been suggested that the VF may be induced and maintained by rotors anchored in specific anatomic areas such as the papillary muscles, ${ }^{17,18}$ and the involvement of these areas in the necrotic process would depend on the location of the infarct. Second, the MI may induce changes in the basic electrophysiological properties of the myocardium that surrounds the recording electrode, and this effect could modify the VF spectral characteristics irrespective of the intrinsic properties of the arrhythmia. Our results support this mechanism, since the location of the infarct induces similar changes in the frequency distri- bution of the EGMs during SR. Specifically, the effect on the power of the low and intermediate frequency bands is very close to the one observed during VF. The differences of the spectral envelope seen in the region above $30 \mathrm{~Hz}$ might be due to the higher conduction velocity of the electric impulse during sinus rhythm.

The effects of the location of the infarcted area on the VF spectral pattern have not been evaluated in humans. Our results suggest that patients with inferior MI show a greater fractional power at the $\mathrm{f}_{\mathrm{d}}$ component. In contrast, the normalized power at the harmonic frequencies is lower when compared to the anterior MIs. The reasons for these differences are unknown. A similar effect is observed in our two-dimensional computer model of ventricular activation when the distance between the infarcted region and the electrode decreases, or when the size of the simulated infarcted area is increased. Inferior MIs can involve the right ventricle and might be closer to the ICD recording electrode than anterior infarcts, potentially explaining the obtained results. In contrast, a larger amount of electrically active myocardium would be expected in the inferior infarct group, since anterior MIs are more often associated with extensive scars. More precise information on the size and location of the infarcted areas as well as their anatomic relationship with the electrode position might be obtained with image techniques and could be useful to better assess this issue, but we lack this information due to the retrospective character of the study. A second limitation derives from the use of an ICD to obtain the EGMs, which reduces the available signal to a unique intracardiac recording. Multiple recordings from both ventricular chambers during sinus rhythm and VF might be useful to further delineate the influence of the MI location and size on the spectral characteristics of the arrhythmia. Finally, the two-dimensional mathematical model of propagation clearly oversimplifies the phenomena involved in the generation of the EGM in an infarcted human heart. It was used only to simulate the effects of the size of the necrotic region and its distance to the recording electrode on the spectrum of the electrical signal. A more realistic approach would require a substantial increase in the complexity of the model and lies beyond the scope of this study.

\section{Conclusion}

The spectral characteristics of the intracardiac signal during VF depend on the MI location. A higher fractional energy in the lowfrequency region is seen in inferior MIs, whereas the peak power at the harmonics increases in anterior infarcts. A similar effect is seen during SR and VF, suggesting that it is caused by local 


\section{SPECTRUM OF MYOCARDIAL INFARCTION DURING VF}

electrophysiologic abnormalities induced by the MI rather than by different intrinsic characteristics of the VF. The mathematical model analysis suggests that this effect could be due either to differences in the position of the electrode relative to the necrotic area or to their separation distance, but further studies considering detailed anatomical information are required to confirm this result. Overall, these results should be taken into account

\section{References}

1. Gray RA, Pertsov AM, Jalife J. Spatial and temporal organization during cardiac fibrillation [published erratum appears in Nature. 1998;393:191]. Nature 1998; 392:75-78.

2. Rogers JM, Huang J, Smith WM, Ideker RE. Incidence, evolution, and spatial distribution of functional reentry during ventricular fibrillation in pigs. Circ Res 1999; 84:945-954.

3. Zaitsev AV, Berenfeld O, Mironov SF, Jalife J, Pertsov AM. Distribution of excitation frequencies on the epicardial and endocardial surfaces of fibrillating ventricular wall of the sheep heart. Circ Res 2000; 86:408-417.

4. Tovar $\mathrm{OH}$, Jones JL. Electrophysiological deterioration during long-duration ventricular fibrillation. Circulation 2000; 102:28862891.

5. Mandapati R, Asano Y, Baxter WT, Gray R, Davidenko J, Jalife J. Quantification of effects of global ischemia on dynamics of ventricular fibrillation in isolated rabbit heart. Circulation 1998; 98:16881696.

6. Chorro FJ, Cánoves J, Guerrero J, Mainar L, Sanchis J, Such L, López-Merino V. Alteration of ventricular fibrillation by flecainide, verapamil, and sotalol: An experimental study. Circulation 2000; 101:1606-1615.

7. Eftestøl T, Sunde K, Aase SO, Husøy JH, Steen PA. Predicting outcome of defibrillation by spectral characterization and nonparametric classification of ventricular fibrillation in patients with out-of-hospital cardiac arrest. Circulation 2000; 102:15231529.

8. Jacobson JT, Johnson D, Norvath G, Goldberger A, Kadish A. Effect of underlying heart disease on the frequency content of ventricular fibrillation in the dog heart. Pacing Clin Electrophysiol 2000; 23:243-252.

9. Kim Y, Xie F, Yashima M, Wu TJ, Valderrábano M, Lee MH, Ohara $\mathrm{T}$, et al. Role of papillary muscle in the generation and maintenance when analyzing further clinical studies involving patients with infarctions in terms of spectral features of their presenting arrhythmias.

Acknowledgments: This work has been partially supported by Research Grant from Boston Scientific, and by Research Project TEC2007-68096-C02/TCM from Spanish Ministerio de Educación y Ciencia. JRC is supported by Grant 01836-04 from Consejería de Educación of Comunidad de Madrid.

of reentry during ventricular tachycardia and fibrillation in isolated swine right ventricle. Circulation 1999; 100:1450-1459.

10. Laguna P, Sörnmo L. Bioelectrical Signal Processing in Cardiac and Neurological Applications. Amsterdam, Academic Press, 2005, p. 700 .

11. Berenfeld O. Quantifying activation frequency in atrial fibrillation to establish underlying mechanisms and ablation guidance. Heart Rhythm 2007; 9:1225-1234.

12. Everett TH, Kok LC, Vaughn RH, Moorman JR, Haines DE. Frequency domain algorithm for quantifying atrial fibrillation organization to increase defibrillation efficacy. IEEE Trans Biomed Eng 2001; 48:969-978.

13. Picone JW. Signal modelling techniques in speech recognition. Proc IEEE 1993; 81:1215-1247.

14. Alonso F, Requena J, García-Alberola A, Rojo JL, Sánchez-Muñoz JJ, Martínez- Sánchez J, Valdés M. A probabilistic model of cardiac electrical activity based on a cellular automata system. Rev Esp Cardiol 2005; 58:41-47.

15. Malmivuo J, Plonsey R. Bioelectromagnetism. Principles and Applications of Bioelectric and Biomagnetic Fields. New York, Oxford University Press, 1995, p. 700.

16. Damle RS, Robinson NS, Ye D, Roth SI, Greene R, Goldberger JJ, Kadish AH. Electrical activation during ventricular fibrillation in the subacute and chronic phases of healing canine myocardial infarction. Circulation 1995; 92:535-545.

17. Thomas SP, Thiagalingam A, Wallace E, Kovoor P, Ross DL. Organization of myocardial activation during ventricular fibrillation after myocardial infarction: Evidence for sustained high-frequency sources. Circulation 2005; 112:157-163.

18. Wu TJ, Lin SF, Baher A, Qu A, Garfinkel A, Weiss JN, Ting CT, et al. Mother rotors and the mechanisms of D600-induced type 2 ventricular fibrillation. Circulation 2004; 110:2110-2118. 